\title{
Forced oscillation of fractional differential equations via conformable derivatives with damping term
}

\author{
Aphirak Aphithana ${ }^{1}$, Sotiris K. Ntouyas ${ }^{2,3}$ and Jessada Tariboon ${ }^{1 *}$ (D)
}

*Correspondence:

jessada.t@sci.kmutnb.ac.th

${ }^{1}$ Intelligent and Nonlinear Dynamic

Innovations Research Center

Department of Mathematics,

Faculty of Applied Science, King

Mongkut's University of Technology

North Bangkok, Bangkok, Thailand

Full list of author information is

available at the end of the article

\begin{abstract}
Based on the properties of nonlocal fractional calculus generated by conformable derivatives, we establish some sufficient conditions for oscillation of all solutions for fractional differential equations with damping term. Forced oscillation of conformable differential equations in the frame of Riemann, as well as of Caputo type, is established. Examples are provided to demonstrate the effectiveness of the main results.
\end{abstract}

MSC: 34A08; 34C10; 26A33

Keywords: Forced oscillation; Oscillation theory; Fractional differential equations; Fractional conformable integrals; Fractional conformable derivatives; Damping

\section{Introduction}

Fractional differential equations gained considerable importance due to their various applications in viscoelasticity, electroanalytical chemistry, control theory, many physical problems, etc. The books [1-6] summarize and organize much of fractional calculus and many of theories and applications of fractional differential equations. Many authors have studied the existence and uniqueness of solutions for different types of fractional boundary value problems; see the papers [7-18] and the references cited therein.

The oscillation theory for fractional differential and difference equations has been studied by some authors (see [19-29]). In [23] the authors studied the oscillation theory for fractional differential equations by considering fractional initial value problem of the form

$$
\left\{\begin{array}{l}
\mathcal{D}_{a}^{q} x(t)+f_{1}(t, x)=v(t)+f_{2}(t, x), \quad t>a, \\
\lim _{t \rightarrow a+} J_{a}^{1-q} x(t)=b,
\end{array}\right.
$$

where $\mathcal{D}_{a}^{q}$ denotes the Riemann-Liouville fractional derivative starting at a point $a$, of order $q$ with $0<q \leq 1, J_{a}^{1-q}$ is the Riemann-Liouville fractional integral starting at a point $a$, of order $1-q, f_{1}, f_{2}$ are continuous functions.

(c) The Author(s) 2019. This article is distributed under the terms of the Creative Commons Attribution 4.0 International License (http://creativecommons.org/licenses/by/4.0/), which permits unrestricted use, distribution, and reproduction in any medium, provided you give appropriate credit to the original author(s) and the source, provide a link to the Creative Commons license, and indicate if changes were made. 
Recently, in [21] the authors studied the oscillation of a conformable initial value problem of the form

$$
\left\{\begin{array}{l}
a^{\mathfrak{D}^{\alpha, \rho}} x(t)+f_{1}(t, x)=r(t)+f_{2}(t, x), \quad t>a, \\
\lim _{t \rightarrow a^{+}} a \mathfrak{J}^{j-\alpha, \rho} x(t)=b_{j} \quad(j=1,2, \ldots, m),
\end{array}\right.
$$

where $m=\lceil\alpha\rceil=\min \{m \in \mathbb{Z} \mid m \geq \alpha\},{ }_{a} \mathfrak{D}^{\alpha, \rho}$ is the left conformable derivative of order $\alpha \in \mathbb{C}, \mathfrak{R}(\alpha) \geq 0$ in the Riemann-Liouville setting and $\mathfrak{J}^{\alpha, \rho}$ is the left conformable integral operator.

In [22] the authors studied forced oscillatory properties of solutions to the nonlinear fractional initial value problem with damping

$$
\left\{\begin{array}{l}
\left(\mathcal{D}_{0^{+}}^{1+\alpha} y\right)(t)+p(t)\left(\mathcal{D}_{0^{+}}^{\alpha} y\right)(t)+q(t) f(y(t))=g(t), \quad t>0, \\
\left(I_{0^{+}}^{1-\alpha} y\right)(0+)=b
\end{array}\right.
$$

where $b$ is a real number, $\alpha \in(0,1)$ is a given constant, and $\mathcal{D}_{0^{+}}^{\alpha}$ is the Riemann-Liouville fractional derivative of order $\alpha$.

In this paper, motivated by the above papers, we study forced oscillatory properties of solutions to the conformable initial value problem with damping in the Riemann-Liouville setting as follows:

$$
\left\{\begin{array}{l}
\mathfrak{D}^{1+\alpha, \rho} x(t)+p(t)_{a} \mathfrak{D}^{\alpha, \rho} x(t)+q(t) f(x(t))=g(t), \quad t>a, \\
\lim _{t \rightarrow a^{+} a} \mathfrak{I}^{j-\alpha, \rho} x(t)=b_{j} \quad(j=1,2, \ldots, m),
\end{array}\right.
$$

where $m=\lceil\alpha\rceil, 0<\rho \leq 1, p \in \mathbb{C}\left(\mathbb{R}^{+}, \mathbb{R}\right), q \in \mathbb{C}\left(\mathbb{R}^{+}, \mathbb{R}^{+}\right), g \in \mathbb{C}\left(\mathbb{R}^{+}, \mathbb{R}\right), f \in \mathbb{C}(\mathbb{R}, \mathbb{R})$ are continuous functions, ${ }_{a} \mathfrak{D}^{\alpha, \rho}$ is the left conformable derivative of order $\alpha \in \mathbb{C}, \mathfrak{R}(\alpha) \geq 0$ in the Riemann-Liouville setting, and ${ }_{a} \mathfrak{I}^{\alpha, \rho}$ is the left conformable integral operator.

Moreover, we study the forced oscillation of conformable initial value problems in the Caputo setting of the form

$$
\left\{\begin{array}{l}
{ }_{a}^{C} \mathfrak{D}^{1+\alpha, \rho} x(t)+p(t){ }_{a}^{C} \mathfrak{D}^{\alpha, \rho} x(t)+q(t) f(x(t))=g(t), \quad t>a, \\
{ }_{a}^{k} D^{\rho} x(a)=b_{k} \quad(k=0,1, \ldots, m-1),
\end{array}\right.
$$

where $m=\lceil\alpha\rceil$, and ${ }_{a}^{C} \mathfrak{D}^{\alpha, \rho} x$ is the left conformable derivative of order $\alpha \in \mathbb{C}, \Re(\alpha) \geq 0$ in the Caputo setting.

Definition 1.1 The solution $x$ of problem (1.4) (respectively (1.5)) is called oscillatory if it is neither eventually positive nor eventually negative. Otherwise, it is called nonoscillatory.

This paper is organized as follows. Section 2 introduces some notations and provides the definitions of conformable fractional integral and differential operators together with some basic properties and lemmas that are needed in the proofs of the main theorems. In Sect. 3, forced oscillation of conformable fractional differential equations in the frame of Riemann is presented, while in Sect. 4 forced oscillation of conformable fractional differential equations in the frame of Caputo is established. Examples are provided in Sect. 5 to demonstrate the effectiveness of the main theorems. 


\section{Preliminaries}

The left conformable derivative starting from $a$ of a function $f:[a, \infty) \rightarrow \mathbb{R}$ of order $0<$ $\rho \leq 1$ is defined by

$$
\left({ }_{a} D^{\rho} f\right)(t)=\lim _{\epsilon \rightarrow 0} \frac{f\left(t+\epsilon(t-a)^{1-\rho}\right)-f(t)}{\epsilon} .
$$

If $\left({ }_{a} D^{\rho} f\right)(t)$ exists on $(a, b)$, then $\left({ }_{a} D^{\rho} f\right)(a)=\lim _{t \rightarrow a^{+}}\left({ }_{a} D^{\rho} f\right)(t)$. If $f$ is differentiable, then

$$
\left({ }_{a} D^{\rho} f\right)(t)=(t-a)^{1-\rho} f^{\prime}(t)
$$

The corresponding left conformable integral is defined as

$$
{ }_{a} I^{\rho} f(x)=\int_{a}^{x} f(t) \frac{d t}{(t-a)^{1-\rho}}, \quad 0<\rho \leq 1
$$

For the extension to the higher order $\rho>1$, see [30].

Definition 2.1 ([31]) The left conformable integral operator is defined by

$$
{ }_{a} \mathfrak{I}^{\alpha, \rho} f(x)=\frac{1}{\Gamma(\alpha)} \int_{a}^{x}\left(\frac{(x-a)^{\rho}-(t-a)^{\rho}}{\rho}\right)^{\alpha-1} \frac{f(t) d t}{(t-a)^{1-\rho}}
$$

where $\alpha \in \mathbb{C}, \Re(\alpha) \geq 0$.

Definition 2.2 ([31]) The left conformable derivative of order $\alpha \in \mathbb{C}, \Re(\alpha) \geq 0$ in the Riemann-Liouville setting is defined by

$$
\begin{aligned}
a_{a} \mathfrak{D}^{\alpha, \rho} f(x) & ={ }_{a}^{m} D^{\rho}\left({ }_{a} \mathfrak{I}^{m-\alpha, \rho}\right) f(x) \\
& =\frac{{ }_{a}^{m} D^{\rho}}{\Gamma(m-\alpha)} \int_{a}^{x}\left(\frac{(x-a)^{\rho}-(t-a)^{\rho}}{\rho}\right)^{m-\alpha-1} \frac{f(t) d t}{(t-a)^{1-\rho}},
\end{aligned}
$$

where $m=\lceil\Re(\alpha)\rceil,{ }_{a}^{m} D^{\rho}=\underbrace{{ }_{a} D^{\rho} D^{\rho} \cdots_{a} D^{\rho}}_{m \text { times }}$, and ${ }_{a} D^{\rho} f$ is the left conformable differential operator presented in (2.1).

Definition 2.3 ([31]) The left Caputo conformable derivative of order $\alpha \in \mathbb{C}, \Re(\alpha) \geq 0$ is defined by

$$
\begin{aligned}
{ }_{a}^{C} \mathfrak{D}^{\alpha, \rho} f(x) & ={ }_{a} \mathfrak{J}^{m-\alpha, \rho}\left({ }_{a}^{m} D^{\rho} f(x)\right) \\
& =\frac{1}{\Gamma(m-\alpha)} \int_{a}^{x}\left(\frac{(x-a)^{\rho}-(t-a)^{\rho}}{\rho}\right)^{m-\alpha-1} \frac{{ }_{a}^{m} D^{\rho} f(t) d t}{(t-a)^{1-\rho}},
\end{aligned}
$$

where $m=\lceil\Re(\alpha)\rceil,{ }_{a}^{m} D^{\rho}=\underbrace{{ }_{a} D_{a} D^{\rho} \cdots{ }_{a} D^{\rho}}_{m \text { times }}$, and ${ }_{a} D^{\rho} f$ is the left conformable differential operator presented in (2.1). 
Lemma 2.1 ([31]) Let $\alpha \in \mathbb{C}$ and ${ }_{a} \mathfrak{I}^{j-\alpha, \rho} x(t)$ be the conformable integral (2.2) of order $j-\alpha$, then

$$
{ }_{a} \mathfrak{I}^{\alpha, \rho}\left({ }_{a} \mathfrak{D}^{\alpha, \rho} f(x)\right)=f(x)-\sum_{j=1}^{m} \frac{{ }_{a} \mathfrak{J}^{j-\alpha, \rho} f(a)}{\rho^{\alpha-j} \Gamma(\alpha-j+1)}(x-a)^{\rho(\alpha-j)} .
$$

\section{Forced oscillation of conformable differential equations in the frame of Riemann}

In this section we study the oscillation theory for equation (1.4). We prove our result under the following assumption:

(H) $p \in \mathbb{C}\left(\mathbb{R}^{+}, \mathbb{R}\right), q \in \mathbb{C}\left(\mathbb{R}^{+}, \mathbb{R}^{+}\right), g \in \mathbb{C}\left(\mathbb{R}^{+}, \mathbb{R}\right), f \in \mathbb{C}(\mathbb{R}, \mathbb{R})$ and $f(u) / u>0$ for all $u \neq 0$.

We set

$$
\Phi(t)=\Gamma(\alpha) \sum_{j=1}^{m} \frac{b_{j}(t-a)^{\rho(\alpha-j)}}{\rho^{\alpha-j} \Gamma(\alpha-j+1)}
$$

and

$$
\Lambda(t, T)=\int_{a}^{T}\left(\frac{(t-a)^{\rho}-(w-a)^{\rho}}{\rho}\right)^{\alpha-1}\left(\frac{M+{ }_{t_{1}} I^{\rho}(g(w) V(w))}{V(w)}\right) \frac{d w}{(w-a)^{1-\rho}}
$$

for $a \leq t \leq T$, where $M={ }_{a} \mathfrak{D}^{\alpha, \rho} x\left(t_{1}\right) V\left(t_{1}\right)$.

Theorem 3.1 Suppose that $(H)$ and for every sufficiently large $T$ the following conditions hold:

$$
\lim _{t \rightarrow \infty} \inf \left(\frac{t^{\rho}}{\rho}\right)^{1-\alpha} \int_{T}^{t}\left(\frac{(t-a)^{\rho}-(w-a)^{\rho}}{\rho}\right)^{\alpha-1}\left(\frac{M+{ }_{t_{1}} I^{\rho}(g(w) V(w))}{V(w)}\right) \frac{d w}{(w-a)^{1-\rho}}=-\infty
$$

and

$$
\lim _{t \rightarrow \infty} \sup \left(\frac{t^{\rho}}{\rho}\right)^{1-\alpha} \int_{T}^{t}\left(\frac{(t-a)^{\rho}-(w-a)^{\rho}}{\rho}\right)^{\alpha-1}\left(\frac{M+{ }_{t_{1}} I^{\rho}(g(w) V(w))}{V(w)}\right) \frac{d w}{(w-a)^{1-\rho}}=\infty,
$$

where $V(t)=\exp \int_{t_{1}}^{t}(s-a)^{\rho-1} p(s) d s, t_{1}>a$, and $M$ is an arbitrary constant. Then every solution of problem (1.4) is oscillatory.

Proof Let $x$ be a nonoscillatory solution of problem (1.4). Without loss of generality, suppose that $T>a$ is large enough and $t_{1}>T$ so that $x(t)>0$ for $t>t_{1}$. According to (1.4) and $(H)$, the following inequality is satisfied:

$$
\begin{aligned}
{ }_{a} D^{\rho}\left[{ }_{a} \mathfrak{D}^{\alpha, \rho} x(t) V(t)\right] & =(t-a)^{1-\rho} \frac{d}{d t}\left[{ }_{a} \mathfrak{D}^{\alpha, \rho} x(t) V(t)\right] \\
& =(t-a)^{1-\rho} \frac{d}{d t}\left({ }_{a} \mathfrak{D}^{\alpha, \rho} x(t)\right) V(t)+(t-a)^{1-\rho}{ }_{a} \mathfrak{D}^{\alpha, \rho} x(t) \frac{d}{d t}(V(t))
\end{aligned}
$$




$$
\begin{aligned}
& ={ }_{a} \mathfrak{D}^{\alpha+1, \rho} x(t) V(t)+p(t)_{a} \mathfrak{D}^{\alpha, \rho} x(t) V(t) \\
& =-q(t) f(x(t)) V(t)+g(t) V(t) \\
& <g(t) V(t) .
\end{aligned}
$$

Taking the left conformable integral order $\rho$ for the above inequality from $t_{1}$ to $t$, we can obtain

$$
\begin{aligned}
{ }_{a} \mathfrak{D}^{\alpha, \rho} x(t) V(t) & <{ }_{a} \mathfrak{D}^{\alpha, \rho} x\left(t_{1}\right) V\left(t_{1}\right)+{ }_{{ }_{1}} I^{\rho}(g(t) V(t)) \\
& =M+{ }_{t_{1}} I^{\rho}(g(t) V(t)) .
\end{aligned}
$$

From Lemma 2.1 and (3.5) we get

$$
{ }_{a} \mathfrak{D}^{\alpha, \rho} x(t)<\frac{M+{ }_{t_{1}} I^{\rho}(g(t) V(t))}{V(t)},
$$

which leads to

$$
x(t)-\sum_{j=1}^{m} \frac{{ }_{a} \mathfrak{I}^{j-\alpha, \rho} x(a)(t-a)^{\rho(\alpha-j)}}{\rho^{\alpha-j} \Gamma(\alpha-j+1)}<{ }_{a} \mathfrak{I}^{\alpha, \rho}\left[\frac{M+{ }_{t_{1}} I^{\rho}(g(t) V(t))}{V(t)}\right] .
$$

So, we have

$$
\begin{aligned}
x(t)< & \sum_{j=1}^{m} \frac{b_{j}(t-a)^{\rho(\alpha-j)}}{\rho^{\alpha-j} \Gamma(\alpha-j+1)} \\
& +\frac{1}{\Gamma(\alpha)} \int_{a}^{t}\left(\frac{(t-a)^{\rho}-(w-a)^{\rho}}{\rho}\right)^{\alpha-1}\left(\frac{M+{ }_{t_{1}} I^{\rho}(g(w) V(w))}{V(w)}\right) \frac{d w}{(w-a)^{1-\rho}}
\end{aligned}
$$

for every sufficiently large $T$. Multiplying both sides of the above inequality by $\Gamma(\alpha)$, we can obtain

$$
\begin{aligned}
\Gamma(\alpha) x(t)< & \Gamma(\alpha) \sum_{j=1}^{m} \frac{b_{j}(t-a)^{\rho(\alpha-j)}}{\rho^{\alpha-j} \Gamma(\alpha-j+1)} \\
& +\int_{a}^{T}\left(\frac{(t-a)^{\rho}-(w-a)^{\rho}}{\rho}\right)^{\alpha-1}\left(\frac{M+{ }_{t_{1}} I^{\rho}(g(w) V(w))}{V(w)}\right) \frac{d w}{(w-a)^{1-\rho}} \\
& +\int_{T}^{t}\left(\frac{(t-a)^{\rho}-(w-a)^{\rho}}{\rho}\right)^{\alpha-1}\left(\frac{M+{ }_{t_{1}} I^{\rho}(g(w) V(w))}{V(w)}\right) \frac{d w}{(w-a)^{1-\rho}} \\
= & \Phi(t)+\Lambda(t, T) \\
& +\int_{T}^{t}\left(\frac{(t-a)^{\rho}-(w-a)^{\rho}}{\rho}\right)^{\alpha-1}\left(\frac{M+{ }_{t_{1}} I^{\rho}(g(w) V(w))}{V(w)}\right) \frac{d w}{(w-a)^{1-\rho}}
\end{aligned}
$$

where $\Phi$ and $\Lambda$ are defined in (3.1) and (3.2), respectively.

Multiplying (3.6) by $\left(\frac{t^{\rho}}{\rho}\right)^{1-\alpha}$, we get

$$
0<\left(\frac{t^{\rho}}{\rho}\right)^{1-\alpha} \Gamma(\alpha) x(t)
$$




$$
\begin{aligned}
& <\left(\frac{t^{\rho}}{\rho}\right)^{1-\alpha} \Phi(t)+\left(\frac{t^{\rho}}{\rho}\right)^{1-\alpha} \Lambda(t, T) \\
& \quad+\left(\frac{t^{\rho}}{\rho}\right)^{1-\alpha} \int_{T}^{t}\left(\frac{(t-a)^{\rho}-(w-a)^{\rho}}{\rho}\right)^{\alpha-1}\left(\frac{M+{ }_{t_{1}} I^{\rho}(g(w) V(w))}{V(w)}\right) \frac{d w}{(w-a)^{1-\rho}} .
\end{aligned}
$$

Taking $T_{1}>T$, we consider two cases as follows.

Case (1): Let $0<\alpha \leq 1$. Then $m=1$ and $\left(\frac{t^{\rho}}{\rho}\right)^{1-\alpha} \Phi(t)=b_{1} t^{\rho-\rho \alpha}(t-a)^{\rho \alpha-\rho}$. Since the function $h_{1}(t)=t^{\rho-\rho \alpha}(t-a)^{\rho \alpha-\rho}$ is decreasing for $\rho>0$ and $\alpha<1$, we get for $t \geq T_{1}$ (see [21])

$$
\left|\left(\frac{t^{\rho}}{\rho}\right)^{1-\alpha} \Phi(t)\right| \leq\left|b_{1}\right| T_{1}^{\rho-\rho \alpha}\left(T_{1}-a\right)^{\rho \alpha-\rho}:=c_{1}\left(T_{1}\right) .
$$

The function $h_{2}(t)=t^{\rho-\rho \alpha}\left[(t-a)^{\rho}-(w-a)^{\rho}\right]^{\alpha-1}$ is decreasing for $\rho>0$ and $\alpha<1$. Thus, we get

$$
\begin{aligned}
& \left|\left(\frac{t^{\rho}}{\rho}\right)^{1-\alpha} \Lambda(t, T)\right| \\
& \quad=\left|\left(\frac{t^{\rho}}{\rho}\right)^{1-\alpha} \int_{a}^{T}\left(\frac{(t-a)^{\rho}-(w-a)^{\rho}}{\rho}\right)^{\alpha-1}\left(\frac{M+{ }_{t_{1}} I^{\rho}(g(w) V(w))}{V(w)}\right) \frac{d w}{(w-a)^{1-\rho}}\right| \\
& \quad \leq \int_{a}^{T}\left(\frac{t^{\rho}}{\rho}\right)^{1-\alpha}\left(\frac{(t-a)^{\rho}-(w-a)^{\rho}}{\rho}\right)^{\alpha-1}\left|\frac{M+{ }_{t_{1}} I^{\rho}(g(w) V(w))}{V(w)}\right| \frac{d w}{(w-a)^{1-\rho}} \\
& \quad \leq \int_{a}^{T}\left(\frac{T_{1}^{\rho}}{\rho}\right)^{1-\alpha}\left(\frac{\left(T_{1}-a\right)^{\rho}-(w-a)^{\rho}}{\rho}\right)^{\alpha-1}\left|\frac{M+{ }_{t_{1}} I^{\rho}(g(w) V(w))}{V(w)}\right| \frac{d w}{(w-a)^{1-\rho}} \\
& \quad:=c_{2}\left(T, T_{1}\right) .
\end{aligned}
$$

Then, from equation (3.7) and $t \geq T_{1}$, we get

$$
\begin{aligned}
& \left(\frac{t^{\rho}}{\rho}\right)^{1-\alpha} \int_{T}^{t}\left(\frac{(t-a)^{\rho}-(w-a)^{\rho}}{\rho}\right)^{\alpha-1}\left(\frac{M+{ }_{t_{1}} I^{\rho}(g(w) V(w))}{V(w)}\right) \frac{d w}{(w-a)^{1-\rho}} \\
& \geq-\left[c_{1}\left(T_{1}\right)+c_{2}\left(T, T_{1}\right)\right],
\end{aligned}
$$

hence

$$
\begin{aligned}
& \lim _{t \rightarrow \infty} \inf \left(\frac{t^{\rho}}{\rho}\right)^{1-\alpha} \int_{T}^{t}\left(\frac{(t-a)^{\rho}-(w-a)^{\rho}}{\rho}\right)^{\alpha-1}\left(\frac{M+{ }_{t_{1}} I^{\rho}(g(w) V(w))}{V(w)}\right) \frac{d w}{(w-a)^{1-\rho}} \\
& \quad \geq-\left[c_{1}\left(T_{1}\right)+c_{2}\left(T, T_{1}\right)\right] \\
& \quad>-\infty
\end{aligned}
$$

which is a contradiction to condition (3.3).

Case (2): Let $\alpha>1$. Then $m \geq 2$. Also $\left(\frac{t-a}{t}\right)^{\rho \alpha-\rho}<1$ for $\alpha>1$ and $\rho>0$. The function $h_{3}(t)=(t-a)^{\rho-\rho j}$ is decreasing for $j>1$ and $\rho>0$. Thus, for $t \geq T_{1}$, we have (see [21])

$$
\left|\left(\frac{t^{\rho}}{\rho}\right)^{1-\alpha} \Phi(t)\right| \leq \Gamma(\alpha) \sum_{j=1}^{m} \frac{\left|b_{j}\right|\left(T_{1}-a\right)^{\rho-\rho j}}{\rho^{1-j} \Gamma(\alpha-j+1)}:=c_{3}\left(T_{1}\right)
$$


Also, since $\left(\frac{t^{\rho}}{\rho}\right)^{1-\alpha}<1$ and $\left(\frac{(t-a)^{\rho}-(w-a)^{\rho}}{t^{\rho}}\right)^{\alpha-1}<1$ for $\alpha>1$ and $\rho>0$, we get

$$
\begin{aligned}
& \left|\left(\frac{t^{\rho}}{\rho}\right)^{1-\alpha} \Lambda(t, T)\right| \\
& \quad=\left|\left(\frac{t^{\rho}}{\rho}\right)^{1-\alpha} \int_{a}^{T}\left(\frac{(t-a)^{\rho}-(w-a)^{\rho}}{\rho}\right)^{\alpha-1}\left(\frac{M+t_{1} I^{\rho}(g(w) V(w))}{V(w)}\right) \frac{d w}{(w-a)^{1-\rho}}\right| \\
& \quad \leq \int_{a}^{T}\left(\frac{(t-a)^{\rho}-(w-a)^{\rho}}{t^{\rho}}\right)^{\alpha-1}\left|\frac{M+{ }_{t_{1}} I^{\rho}(g(w) V(w))}{V(w)}\right| \frac{d w}{(w-a)^{1-\rho}} \\
& \quad \leq \int_{a}^{T}\left|\left(\frac{M+{ }_{t_{1}} I^{\rho}(g(w) V(w))}{V(w)}\right)\right| \frac{d w}{(w-a)^{1-\rho}}:=c_{4}(T) .
\end{aligned}
$$

From (3.7), (3.10), and (3.11), we conclude that

$$
\begin{aligned}
& \left(\frac{t^{\rho}}{\rho}\right)^{1-\alpha} \int_{T}^{t}\left(\frac{(t-a)^{\rho}-(w-a)^{\rho}}{\rho}\right)^{\alpha-1}\left(\frac{M+{ }_{t_{1}} I^{\rho}(g(w) V(w))}{V(w)}\right) \frac{d w}{(w-a)^{1-\rho}} \\
& \geq-\left[c_{3}\left(T_{1}\right)+c_{4}(T)\right]
\end{aligned}
$$

for $t \geq T_{1}$. Hence

$$
\begin{aligned}
& \lim _{t \rightarrow \infty} \inf \left(\frac{t^{\rho}}{\rho}\right)^{1-\alpha} \int_{T}^{t}\left(\frac{(t-a)^{\rho}-(w-a)^{\rho}}{\rho}\right)^{\alpha-1}\left(\frac{M+{ }_{t_{1}} I^{\rho}(g(w) V(w))}{V(w)}\right) \frac{d w}{(w-a)^{1-\rho}} \\
& \quad \geq-\left[c_{3}\left(T_{1}\right)+c_{4}(T)\right] \\
& \quad>-\infty
\end{aligned}
$$

which is a contradiction to condition (3.3). Therefore, we get that $x(t)$ is oscillatory. In case $x$ is eventually negative, similar arguments lead to a contradiction with condition (3.4). The proof is completed.

\section{Forced oscillation of conformable differential equations in the frame of \\ Caputo}

In this section, we study the forced oscillation of conformable initial value problem (1.5).

We set

$$
\Psi(t)=\Gamma(\alpha) \sum_{k=0}^{m-1} \frac{b_{k}(t-a)^{\rho k}}{\rho^{k} k !}
$$

and

$$
\Omega(t, T)=\int_{a}^{T}\left(\frac{(t-a)^{\rho}-(w-a)^{\rho}}{\rho}\right)^{\alpha-1}\left(\frac{M^{*}+{ }_{t_{1}} I^{\rho}(g(w) V(w))}{V(w)}\right) \frac{d w}{(w-a)^{1-\rho}}
$$

for $a \leq t \leq T$, where $M^{*}={ }_{a}^{C} \mathfrak{D}^{\alpha, \rho} x\left(t_{1}\right) V\left(t_{1}\right)$.

Lemma 4.1 [31] Let $f \in C_{\rho, a}^{m}[a, b]$ and $\alpha \in \mathbb{C}$, then

$$
{ }_{a} \mathfrak{I}^{\alpha, \rho}\left({ }_{a}^{C} \mathfrak{D}^{\alpha, \rho} f(x)\right)=f(x)-\sum_{k=0}^{m-1} \frac{{ }_{a}^{k} D^{\rho} f(a)(x-a)^{\rho k}}{\rho^{k} k !} .
$$


Lemma 4.2 [31] Let $\alpha, \beta \in \mathbb{C}$. If the conformable derivatives ${ }_{a}^{C} \mathfrak{D}^{\alpha, \rho} f(x)$ and ${ }_{a}^{C} \mathfrak{D}^{\alpha+\beta, \rho} f(x)$ exist, then

$$
{ }_{a}^{C} \mathfrak{D}^{\alpha, \rho}\left({ }_{a}^{C} \mathfrak{D}^{\beta, \rho} f(x)\right)={ }_{a}^{C} \mathfrak{D}^{\alpha+\beta, \rho} f(x) .
$$

Lemma 4.3 [31] Let $\alpha \in \mathbb{C}, m=\lceil\Re(\alpha)\rceil$. If $\alpha \in \mathbb{N}$, then

$$
{ }_{a}^{C} \mathfrak{D}^{\alpha, \rho} f(x)={ }_{a}^{m} D^{\rho} f(x) .
$$

Theorem 4.1 Suppose that $(H)$ and for every sufficiently large $T$ the following conditions hold:

$$
\begin{aligned}
& \lim _{t \rightarrow \infty} \inf \left(\frac{t^{\rho}}{\rho}\right)^{1-m} \int_{T}^{t}\left(\frac{(t-a)^{\rho}-(w-a)^{\rho}}{\rho}\right)^{\alpha-1} \\
& \quad \times\left(\frac{M^{*}+{ }_{t_{1}} I^{\rho}(g(w) V(w))}{V(w)}\right) \frac{d w}{(w-a)^{1-\rho}}=-\infty
\end{aligned}
$$

and

$$
\begin{aligned}
& \lim _{t \rightarrow \infty} \sup \left(\frac{t^{\rho}}{\rho}\right)^{1-m} \int_{T}^{t}\left(\frac{(t-a)^{\rho}-(w-a)^{\rho}}{\rho}\right)^{\alpha-1} \\
& \quad \times\left(\frac{M^{*}+{ }_{t_{1}} I^{\rho}(g(w) V(w))}{V(w)}\right) \frac{d w}{(w-a)^{1-\rho}}=\infty,
\end{aligned}
$$

where $V(t)=\exp \int_{t_{1}}^{t}(s-a)^{\rho-1} p(s) d s, t_{1}>a$, and $M^{*}$ is an arbitrary constant. Then every solution of problem (1.5) is oscillatory.

Proof Let $x$ be a nonoscillatory solution of problem (1.5). Without loss of generality, suppose that $T>a$ is large enough and $t_{1}>T$ so that $x(t)>0$ for $t>t_{1}$. According to (1.5) and $(H)$, the following inequality is satisfied:

$$
\begin{aligned}
{ }_{a} D^{\rho}\left[{ }_{a}^{C} \mathfrak{D}^{\alpha, \rho} x(t) V(t)\right] & =(t-a)^{1-\rho} \frac{d}{d t}\left[{ }_{a}^{C} \mathfrak{D}^{\alpha, \rho} x(t) V(t)\right] \\
& =(t-a)^{1-\rho} \frac{d}{d t}\left({ }_{a}^{C} \mathfrak{D}^{\alpha, \rho} x(t)\right) V(t)+(t-a) \stackrel{1-\rho}{a} \mathfrak{D}^{\alpha, \rho} x(t) \frac{d}{d t}(V(t)) \\
& ={ }_{a}^{C} \mathfrak{D}^{\alpha+1, \rho} x(t) V(t)+p(t){ }_{a}^{C} \mathfrak{D}^{\alpha, \rho} x(t) V(t) \\
& =-q(t) f(x(t)) V(t)+g(t) V(t) \\
& <g(t) V(t) .
\end{aligned}
$$

Taking the left conformable integral of order $\rho$ to the above inequality from $t_{1}$ to $t$, we can obtain

$$
\begin{aligned}
{ }_{a}^{C} \mathfrak{D}^{\alpha, \rho} x(t) V(t) & <{ }_{a}^{C} \mathfrak{D}^{\alpha, \rho} x\left(t_{1}\right) V\left(t_{1}\right)+{ }_{t_{1}} I^{\rho}(g(t) V(t)) \\
& =M^{*}+{ }_{{ }_{1}} I^{\rho}(g(t) V(t)) .
\end{aligned}
$$


From Lemma 4.1 and (4.8) we have

$$
{ }_{a}^{C} \mathfrak{D}^{\alpha, \rho} x(t)<\frac{M^{*}+{ }_{{ }_{1}} I^{\rho}(g(t) V(t))}{V(t)} .
$$

Then we get

$$
x(t)-\sum_{k=0}^{m-1} \frac{{ }_{a}^{C} D^{\rho} x(a)(t-a)^{\rho k}}{\rho^{k} k !}<{ }_{a} \mathfrak{I}^{\alpha, \rho}\left[\frac{M^{*}+{ }_{t_{1}} I^{\rho}(g(t) V(t))}{V(t)}\right] .
$$

So, we have

$$
\begin{aligned}
x(t)< & \sum_{k=0}^{m-1} \frac{{ }_{a}^{C} D^{\rho} x(a)(t-a)^{\rho k}}{\rho^{k} k !} \\
& +\frac{1}{\Gamma(\alpha)} \int_{a}^{t}\left(\frac{(t-a)^{\rho}-(w-a)^{\rho}}{\rho}\right)^{\alpha-1}\left(\frac{M^{*}+{ }_{t_{1}} I^{\rho}(g(w) V(w))}{V(w)}\right) \frac{d w}{(w-a)^{1-\rho}}
\end{aligned}
$$

for every sufficiently large $T$. Multiplying both sides of the above inequality by a constant $\Gamma(\alpha)$, we have

$$
\begin{aligned}
\Gamma(\alpha) x(t)< & \Gamma(\alpha) \sum_{k=0}^{m-1} \frac{{ }_{a}^{C} D^{\rho} x(a)(t-a)^{\rho k}}{\rho^{k} k !} \\
& +\int_{a}^{T}\left(\frac{(t-a)^{\rho}-(w-a)^{\rho}}{\rho}\right)^{\alpha-1}\left(\frac{M^{*}+{ }_{t_{1}} I^{\rho}(g(w) V(w))}{V(w)}\right) \frac{d w}{(w-a)^{1-\rho}} \\
& +\int_{T}^{t}\left(\frac{(t-a)^{\rho}-(w-a)^{\rho}}{\rho}\right)^{\alpha-1}\left(\frac{M^{*}+{ }_{t_{1}} I^{\rho}(g(w) V(w))}{V(w)}\right) \frac{d w}{(w-a)^{1-\rho}} \\
= & \Psi(t)+\Omega(t, T) \\
& +\int_{T}^{t}\left(\frac{(t-a)^{\rho}-(w-a)^{\rho}}{\rho}\right)^{\alpha-1}\left(\frac{M^{*}+{ }_{t_{1}} I^{\rho}(g(w) V(w))}{V(w)}\right) \frac{d w}{(w-a)^{1-\rho}}
\end{aligned}
$$

where $\Psi$ and $\Omega$ are defined in (4.1) and (4.2), respectively.

Multiplying (4.9) by $\left(\frac{t^{\rho}}{\rho}\right)^{1-m}$, we get

$$
\begin{aligned}
0< & \left(\frac{t^{\rho}}{\rho}\right)^{1-m} \Gamma(\alpha) x(t) \\
< & \left(\frac{t^{\rho}}{\rho}\right)^{1-m} \Psi(t)+\left(\frac{t^{\rho}}{\rho}\right)^{1-m} \Omega(t, T) \\
& +\left(\frac{t^{\rho}}{\rho}\right)^{1-m} \int_{T}^{t}\left(\frac{(t-a)^{\rho}-(w-a)^{\rho}}{\rho}\right)^{\alpha-1} \\
& \times\left(\frac{M^{*}+{ }_{t_{1}} I^{\rho}(g(w) V(w))}{V(w)}\right) \frac{d w}{(w-a)^{1-\rho}} .
\end{aligned}
$$

Take $T_{1}>T$. We consider two cases as follows. 
Case (1): Let $0<\alpha \leq 1$. Then $m=1$ and $\left(\frac{t^{\rho}}{\rho}\right)^{1-m} \Psi(t)=\Gamma(\alpha) b_{0}$.

The function $h_{4}(t)=\left(\frac{(t-a)^{\rho}-(w-a)^{\rho}}{\rho}\right)^{\alpha-1}$ is decreasing for $\rho>0, t>T_{1}>w$, and $\alpha<1$. Thus, we get

$$
\begin{aligned}
& \left|\left(\frac{t^{\rho}}{\rho}\right)^{1-m} \Omega(t, T)\right| \\
& \quad=\left|\int_{a}^{T}\left(\frac{(t-a)^{\rho}-(w-a)^{\rho}}{\rho}\right)^{\alpha-1}\left(\frac{M^{*}+{ }_{t_{1}} I^{\rho}(g(w) V(w))}{V(w)}\right) \frac{d w}{(w-a)^{1-\rho}}\right| \\
& \quad \leq \int_{a}^{T}\left(\frac{(t-a)^{\rho}-(w-a)^{\rho}}{\rho}\right)^{\alpha-1}\left|\frac{M^{*}+{ }_{t_{1}} I^{\rho}(g(w) V(w))}{V(w)}\right| \frac{d w}{(w-a)^{1-\rho}} \\
& \quad \leq \int_{a}^{T}\left(\frac{\left(T_{1}-a\right)^{\rho}-(w-a)^{\rho}}{\rho}\right)^{\alpha-1}\left|\frac{M^{*}+{ }_{t_{1}} I^{\rho}(g(w) V(w))}{V(w)}\right| \frac{d w}{(w-a)^{1-\rho}} \\
& \quad:=c_{5}\left(T, T_{1}\right) .
\end{aligned}
$$

Then, from equation (4.10) and $t \geq T_{1}$, we get

$$
\begin{aligned}
& \left(\frac{t^{\rho}}{\rho}\right)^{1-m} \int_{T}^{t}\left(\frac{(t-a)^{\rho}-(w-a)^{\rho}}{\rho}\right)^{\alpha-1}\left(\frac{M^{*}+{ }_{t_{1}} I^{\rho}(g(w) V(w))}{V(w)}\right) \frac{d w}{(w-a)^{1-\rho}} \\
& \geq-\left[\Gamma(\alpha) b_{0}+c_{5}\left(T, T_{1}\right)\right],
\end{aligned}
$$

hence

$$
\begin{aligned}
& \lim _{t \rightarrow \infty} \inf \left(\frac{t^{\rho}}{\rho}\right)^{1-m} \int_{T}^{t}\left(\frac{(t-a)^{\rho}-(w-a)^{\rho}}{\rho}\right)^{\alpha-1}\left(\frac{M^{*}+{ }_{t_{1}} I^{\rho}(g(w) V(w))}{V(w)}\right) \frac{d w}{(w-a)^{1-\rho}} \\
& \quad \geq-\left[\Gamma(\alpha) b_{0}+c_{5}\left(T, T_{1}\right)\right] \\
& \quad>-\infty
\end{aligned}
$$

which contradicts condition (4.6).

Case (2): Let $\alpha>1$. Then $m \geq 2$. Also $\left(\frac{t-a}{t}\right)^{\rho m-\rho}<1$ for $m \geq 2$ and $\rho>0$. The function $h_{5}(t)=(t-a)^{\rho(k-m+1)}$ is decreasing for $k<m-1$ and $\rho>0$. Thus, for $t \geq T_{1}$, we have

$$
\begin{aligned}
\left|\left(\frac{t^{\rho}}{\rho}\right)^{1-m} \Psi(t)\right| & =\left|\left(\frac{t^{\rho}}{\rho}\right)^{1-m} \Gamma(\alpha) \sum_{k=0}^{m-1} \frac{b_{k}(t-a)^{\rho k}}{\rho^{k} k !}\right| \\
& =\left|\left(\frac{t-a}{t}\right)^{\rho m-\rho} \Gamma(\alpha) \sum_{k=0}^{m-1} \frac{b_{k}(t-a)^{\rho(k-m+1)}}{\rho^{k-m+1} k !}\right| \\
& \leq \Gamma(\alpha) \sum_{k=0}^{m-1} \frac{\left|b_{k}\right|(t-a)^{\rho(k-m+1)}}{\rho^{k-m+1} k !} \\
& \leq \Gamma(\alpha) \sum_{k=0}^{m-1} \frac{\left|b_{k}\right|\left(T_{1}-a\right)^{\rho(k-m+1)}}{\rho^{k-m+1} k !}:=c_{6}\left(T_{1}\right) .
\end{aligned}
$$


Also, since $\left(\frac{t^{\rho}}{\rho}\right)^{1-m}<1$ and $\left(\frac{(t-a)^{\rho}-(w-a)^{\rho}}{t^{\rho}}\right)^{\alpha-1}<1$ for $\alpha>1$ and $\rho>0$, we get

$$
\begin{aligned}
& \left|\left(\frac{t^{\rho}}{\rho}\right)^{1-m} \Omega(t, T)\right| \\
& \quad=\left|\left(\frac{t^{\rho}}{\rho}\right)^{1-m} \int_{a}^{T}\left(\frac{(t-a)^{\rho}-(w-a)^{\rho}}{\rho}\right)^{\alpha-1}\left(\frac{M^{*}+{ }_{t_{1}} I^{\rho}(g(w) V(w))}{V(w)}\right) \frac{d w}{(w-a)^{1-\rho}}\right| \\
& \quad \leq \int_{a}^{T}\left(\frac{(t-a)^{\rho}-(w-a)^{\rho}}{t^{\rho}}\right)^{\alpha-1}\left|\frac{M^{*}+{ }_{t_{1}} I^{\rho}(g(w) V(w))}{V(w)}\right| \frac{d w}{(w-a)^{1-\rho}} \\
& \quad \leq \int_{a}^{T}\left|\left(\frac{M^{*}+{ }_{t_{1}} I^{\rho}(g(w) V(w))}{V(w)}\right)\right| \frac{d w}{(w-a)^{1-\rho}}:=c_{7}(T) .
\end{aligned}
$$

From (4.10), (4.12), and (4.13), we conclude that

$$
\begin{aligned}
& \left(\frac{t^{\rho}}{\rho}\right)^{1-m} \int_{T}^{t}\left(\frac{(t-a)^{\rho}-(w-a)^{\rho}}{\rho}\right)^{\alpha-1}\left(\frac{M^{*}+{ }_{t_{1}} I^{\rho}(g(w) V(w))}{V(w)}\right) \frac{d w}{(w-a)^{1-\rho}} \\
& \geq-\left[c_{6}\left(T_{1}\right)+c_{7}(T)\right]
\end{aligned}
$$

for $t \geq T_{1}$. Hence

$$
\begin{aligned}
& \lim _{t \rightarrow \infty} \inf \left(\frac{t^{\rho}}{\rho}\right)^{1-m} \int_{T}^{t}\left(\frac{(t-a)^{\rho}-(w-a)^{\rho}}{\rho}\right)^{\alpha-1}\left(\frac{M^{*}+{ }_{t_{1}} I^{\rho}(g(w) V(w))}{V(w)}\right) \frac{d w}{(w-a)^{1-\rho}} \\
& \quad \geq-\left[c_{6}\left(T_{1}\right)+c_{7}(T)\right] \\
& \quad>-\infty
\end{aligned}
$$

which contradicts condition (4.6). Therefore, we conclude that $x$ is oscillatory. In case $x$ is eventually negative, similar arguments lead to a contradiction with condition (4.7). The proof is completed.

\section{Examples}

In this section, we present examples to illustrate our results.

Example 5.1 Consider the conformable initial value problem

$$
\left\{\begin{array}{l}
{ }_{0} \mathfrak{D}^{\frac{3}{2}, 1} x(t)-{ }_{0} \mathfrak{D}^{\frac{1}{2}, 1} x(t)+(t+5)^{2}(2 x+5) e^{\sin 2 x}=e^{2 t} \cos t, \quad t>0, \\
\lim _{t \rightarrow 0^{+}} \mathfrak{I}^{\frac{1}{2}, 1} x(t)=0 .
\end{array}\right.
$$

Here $\alpha=1 / 2, \rho=1, a=0, p(t)=-1, q(t)=(t+5)^{2}, f(x)=(2 x+5) e^{\sin 2 x}, g(t)=e^{2 t} \cos t$, and $V(s)=e^{t_{1}-s}$. It is easy to verify that assumption $(H)$ is satisfied if $x(t)>0$. Then

$$
\begin{aligned}
t_{1} I^{1}(g(w) V(w)) & =\int_{t_{1}}^{w} g(s) V(s) d s \\
& =\int_{t_{1}}^{w} e^{2 s} \cos s e^{t_{1}-s} d s \\
& =\frac{e^{t_{1}+w}}{2}(\sin w+\cos w)-\frac{e^{2 t_{1}}}{2}\left(\sin t_{1}+\cos t_{1}\right)
\end{aligned}
$$




$$
=\frac{e^{t_{1}+w}}{2} \sqrt{2} \sin \left(w+\frac{\pi}{4}\right)-\frac{e^{2 t_{1}}}{2}\left(\sin t_{1}+\cos t_{1}\right) .
$$

Set $t_{1}=\pi / 2$. Hence, we can obtain

$$
\begin{gathered}
\left(\frac{t^{\rho}}{\rho}\right)^{1-\alpha} \int_{0}^{t}\left(\frac{(t-a)^{\rho}-(w-a)^{\rho}}{\rho}\right)^{\alpha-1}\left(\frac{M+{ }_{t_{1}} I^{\rho}(g(w) V(w))}{V(w)}\right) \frac{d w}{(w-a)^{1-\rho}} \\
=t^{\frac{1}{2}} \int_{0}^{t}(t-w)^{-\frac{1}{2}} e^{w-\frac{\pi}{2}}\left(\left(M-\frac{e^{\pi}}{2}\right)+\frac{\sqrt{2}}{2} e^{\frac{\pi}{2}+w} \sin \left(w+\frac{\pi}{4}\right)\right) d w .
\end{gathered}
$$

Set $t-w=s^{2}$, then the above integral can be written as the following form:

$$
\begin{aligned}
& t^{\frac{1}{2}} \int_{\sqrt{t}}^{0} \frac{1}{s} e^{t-s^{2}-\frac{\pi}{2}}\left(\left(\frac{2 M-e^{\pi}}{2}\right)+\frac{\sqrt{2}}{2} e^{\frac{\pi}{2}+t-s^{2}} \sin \left(t-s^{2}+\frac{\pi}{4}\right)\right)(-2 s) d s \\
& =t^{\frac{1}{2}}\left(2 M-e^{\pi}\right) e^{t-\frac{\pi}{2}} \int_{0}^{\sqrt{t}} e^{-s^{2}} d s+t^{\frac{1}{2}} \sqrt{2} e^{2 t} \int_{0}^{\sqrt{t}} e^{-2 s^{2}} \sin \left(t-s^{2}+\frac{\pi}{4}\right) d s \\
& =t^{\frac{1}{2}}\left(2 M-e^{\pi}\right) e^{t-\frac{\pi}{2}} \int_{0}^{\sqrt{t}} e^{-s^{2}} d s+t^{\frac{1}{2}} \sqrt{2} e^{2 t} \sin \left(t+\frac{\pi}{4}\right) \int_{0}^{\sqrt{t}} e^{-2 s^{2}} \cos s^{2} d s \\
& -t^{\frac{1}{2}} \sqrt{2} e^{2 t} \cos \left(t+\frac{\pi}{4}\right) \int_{0}^{\sqrt{t}} e^{-2 s^{2}} \sin s^{2} d s
\end{aligned}
$$

Let $t \rightarrow+\infty$, as the result of $\left|e^{-2 s^{2}} \cos s^{2}\right| \leq e^{-2 s^{2}}, \quad\left|e^{-2 s^{2}} \sin s^{2}\right| \leq e^{-2 s^{2}}$ and $\lim _{t \rightarrow+\infty} \int_{0}^{\sqrt{t}} e^{-2 s^{2}} d s=\frac{\sqrt{2 \pi}}{4}$. So, we know that

$$
\lim _{t \rightarrow+\infty} \int_{0}^{\sqrt{t}} e^{-2 s^{2}} \cos s^{2} d s \text { and } \lim _{t \rightarrow+\infty} \int_{0}^{\sqrt{t}} e^{-2 s^{2}} \sin s^{2} d s
$$

are convergent.

Thus, we can set $\lim _{t \rightarrow+\infty} \int_{0}^{\sqrt{t}} e^{-2 s^{2}} \cos s^{2} d s=A, \lim _{t \rightarrow+\infty} \int_{0}^{\sqrt{t}} e^{-2 s^{2}} \sin s^{2} d s=B$. Select the sequence $\left\{t_{k}\right\}=\left\{\frac{7 \pi}{2}-\frac{\pi}{4}+2 k \pi-\arctan \frac{-B}{A}\right\}, \lim _{k \rightarrow \infty} t_{k}=\infty$, then we calculate the following term:

$$
\begin{aligned}
& \lim _{k \rightarrow \infty}\left\{t _ { k } ^ { \frac { 1 } { 2 } } e ^ { t _ { k } } \left[\left(2 M-e^{\pi}\right) e^{-\frac{\pi}{2}} \int_{0}^{\sqrt{t_{k}}} e^{-s^{2}} d s+\sqrt{2} e^{t_{k}}\left(\sin \left(t_{k}+\frac{\pi}{4}\right) \int_{0}^{\sqrt{t_{k}}} e^{-2 s^{2}} \cos s^{2} d s\right.\right.\right. \\
& \left.\left.\left.\quad-\cos \left(t_{k}+\frac{\pi}{4}\right) \int_{0}^{\sqrt{t_{k}}} e^{-2 s^{2}} \sin s^{2} d s\right)\right]\right\} .
\end{aligned}
$$

Firstly, we consider the following limit:

$$
\begin{aligned}
& \lim _{k \rightarrow \infty}\left(\sin \left(t_{k}+\frac{\pi}{4}\right) \int_{0}^{\sqrt{t_{k}}} e^{-2 s^{2}} \cos s^{2} d s-\cos \left(t_{k}+\frac{\pi}{4}\right) \int_{0}^{\sqrt{t_{k}}} e^{-2 s^{2}} \sin s^{2} d s\right) \\
& =A \cdot \lim _{k \rightarrow \infty} \sin \left(\frac{7 \pi}{2}+2 k \pi-\arctan \frac{-B}{A}\right)-B \cdot \lim _{k \rightarrow \infty} \cos \left(\frac{7 \pi}{2}+2 k \pi-\arctan \frac{-B}{A}\right) \\
& =A \cdot \sin \left(\frac{7 \pi}{2}-\arctan \frac{-B}{A}\right)-B \cdot \cos \left(\frac{7 \pi}{2}-\arctan \frac{-B}{A}\right) \\
& =-\sqrt{A^{2}+B^{2}} .
\end{aligned}
$$


Secondly, we know that $\lim _{k \rightarrow \infty} t_{k}^{\frac{1}{2}} e^{t_{k}}=+\infty$ and $\lim _{k \rightarrow \infty}\left(2 M-e^{\pi}\right) e^{-\frac{\pi}{2}} \int_{0}^{\sqrt{t_{k}}} e^{-s^{2}} d s=(2 M-$ $\left.e^{\pi}\right) e^{-\frac{\pi}{2}} \frac{\sqrt{\pi}}{2}$. Hence, for (5.2), we have

$$
\begin{aligned}
\lim _{k \rightarrow \infty}\left\{t _ { k } ^ { \frac { 1 } { 2 } } e ^ { t _ { k } } \left[\left(2 M-e^{\pi}\right) e^{-\frac{\pi}{2}} \int_{0}^{\sqrt{t_{k}}} e^{-s^{2}} d s+\sqrt{2} e^{t_{k}}\left(\sin \left(t_{k}+\frac{\pi}{4}\right) \int_{0}^{\sqrt{t_{k}}} e^{-2 s^{2}} \cos s^{2} d s\right.\right.\right. \\
\left.\left.\left.\quad-\cos \left(t_{k}+\frac{\pi}{4}\right) \int_{0}^{\sqrt{t_{k}}} e^{-2 s^{2}} \sin s^{2} d s\right)\right]\right\} \\
=(+\infty) \cdot\left[\left(2 M-e^{\pi}\right) e^{-\frac{\pi}{2}} \frac{\sqrt{\pi}}{2}+(+\infty)\left(-\sqrt{A^{2}+B^{2}}\right)\right] \\
=-\infty .
\end{aligned}
$$

Then we obtain

$$
\begin{aligned}
& \lim _{t \rightarrow \infty} \inf \left(\frac{t^{\rho}}{\rho}\right)^{1-\alpha} \int_{0}^{t}\left(\frac{(t-a)^{\rho}-(w-a)^{\rho}}{\rho}\right)^{\alpha-1} \\
& \quad \times\left(\frac{M+{ }_{t_{1}} I^{\rho}(g(w) V(w))}{V(w)}\right) \frac{d w}{(w-a)^{1-\rho}}=-\infty .
\end{aligned}
$$

Similarly, selecting the sequence $\left\{t_{l}\right\}=\left\{\frac{5 \pi}{2}-\frac{\pi}{4}+2 l \pi-\arctan \frac{-B}{A}\right\}$, we can obtain

$$
\lim _{t \rightarrow \infty} \sup \left(\frac{t^{\rho}}{\rho}\right)^{1-\alpha} \int_{0}^{t}\left(\frac{(t-a)^{\rho}-(w-a)^{\rho}}{\rho}\right)^{\alpha-1}\left(\frac{M+{ }_{t_{1}} I^{\rho}(g(w) V(w))}{V(w)}\right) \frac{d w}{(w-a)^{1-\rho}}=\infty
$$

Hence, by Theorem 3.1 all solutions of (5.1) are oscillatory.

Example 5.2 Consider the Caputo conformable initial value problem

$$
\left\{\begin{array}{l}
{ }_{0}^{C} \mathfrak{D}^{\frac{3}{2}}, 1 \\
x(0) \\
x(0)=0 .
\end{array}\right.
$$

Here $\alpha=1 / 2, \rho=1, a=0, m=1, p(t)=-1, q(t)=e^{t^{2}}, f(x)=\ln (x+e), g(t)=e^{2 t} \sin t$, and $V(s)=e^{t_{1}-s}$. Thus assumption $(H)$ is satisfied. Then we have

$$
{ }_{t_{1}} I^{1}(g(w) V(w))=\frac{e^{t_{1}+w}}{2} \sqrt{2} \sin \left(w-\frac{\pi}{4}\right)-\frac{e^{2 t_{1}}}{2}\left(\sin t_{1}-\cos t_{1}\right)
$$

By setting $t_{1}=\pi / 4$ and $t-w=s^{2}$, we obtain

$$
\begin{aligned}
& \left(\frac{t^{\rho}}{\rho}\right)^{1-m} \int_{0}^{t}\left(\frac{(t-a)^{\rho}-(w-a)^{\rho}}{\rho}\right)^{\alpha-1}\left(\frac{M^{*}+{ }_{t_{1}} I^{\rho}(g(w) V(w))}{V(w)}\right) \frac{d w}{(w-a)^{1-\rho}} \\
& =\int_{0}^{t}(t-w)^{-\frac{1}{2}} e^{w-\frac{\pi}{4}}\left(M^{*}+\frac{\sqrt{2}}{2} e^{\frac{\pi}{4}+w} \sin \left(w-\frac{\pi}{4}\right)\right) d w
\end{aligned}
$$

and

$$
\int_{\sqrt{t}}^{0} \frac{1}{s} e^{t-s^{2}-\frac{\pi}{4}}\left(M^{*}+\frac{\sqrt{2}}{2} e^{\frac{\pi}{4}+t-s^{2}} \sin \left(t-s^{2}-\frac{\pi}{4}\right)\right)(-2 s) d s
$$




$$
\begin{aligned}
= & 2 M^{*} e^{t-\frac{\pi}{4}} \int_{0}^{\sqrt{t}} e^{-s^{2}} d s+\sqrt{2} e^{2 t} \sin \left(t-\frac{\pi}{4}\right) \int_{0}^{\sqrt{t}} e^{-2 s^{2}} \cos s^{2} d s \\
& -\sqrt{2} e^{2 t} \cos \left(t-\frac{\pi}{4}\right) \int_{0}^{\sqrt{t}} e^{-2 s^{2}} \sin s^{2} d s,
\end{aligned}
$$

respectively. Using the method in Example 5.1, we choose a sequence

$$
\left\{t_{k}\right\}=\left\{\frac{3 \pi}{2}+\frac{\pi}{4}+2 k \pi-\arctan \frac{-B}{A}\right\},
$$

where the constants $A$ and $B$ are defined in Example 5.1. Then we calculate

$$
\begin{aligned}
& \lim _{k \rightarrow \infty}\left(\sin \left(t_{k}-\frac{\pi}{4}\right) \int_{0}^{\sqrt{t_{k}}} e^{-2 s^{2}} \cos s^{2} d s-\cos \left(t_{k}-\frac{\pi}{4}\right) \int_{0}^{\sqrt{t_{k}}} e^{-2 s^{2}} \sin s^{2} d s\right) \\
& =A \cdot \lim _{k \rightarrow \infty} \sin \left(\frac{3 \pi}{2}+2 k \pi-\arctan \frac{-B}{A}\right)-B \cdot \lim _{k \rightarrow \infty} \cos \left(\frac{3 \pi}{2}+2 k \pi-\arctan \frac{-B}{A}\right) \\
& =-\sqrt{A^{2}+B^{2}}
\end{aligned}
$$

and

$$
\begin{aligned}
\lim _{k \rightarrow \infty}\left\{e ^ { t _ { k } } \left[2 M^{*} e^{-\frac{\pi}{4}} \int_{0}^{\sqrt{t_{k}}} e^{-s^{2}} d s+\sqrt{2} e^{t_{k}}\left(\sin \left(t_{k}-\frac{\pi}{4}\right) \int_{0}^{\sqrt{t_{k}}} e^{-2 s^{2}} \cos s^{2} d s\right.\right.\right. \\
\left.\left.\left.-\cos \left(t_{k}-\frac{\pi}{4}\right) \int_{0}^{\sqrt{t_{k}}} e^{-2 s^{2}} \sin s^{2} d s\right)\right]\right\} \\
=(+\infty) \cdot\left[2 M^{*} e^{-\frac{\pi}{4}} \frac{\sqrt{\pi}}{2}+(+\infty)\left(-\sqrt{A^{2}+B^{2}}\right)\right]=-\infty .
\end{aligned}
$$

Then we obtain

$$
\begin{aligned}
& \lim _{t \rightarrow \infty} \inf \left(\frac{t^{\rho}}{\rho}\right)^{1-m} \int_{0}^{t}\left(\frac{(t-a)^{\rho}-(w-a)^{\rho}}{\rho}\right)^{\alpha-1} \\
& \quad \times\left(\frac{M^{*}+{ }_{t_{1}} I^{\rho}(g(w) V(w))}{V(w)}\right) \frac{d w}{(w-a)^{1-\rho}}=-\infty .
\end{aligned}
$$

Similarly, by selecting the sequence $\left\{t_{l}\right\}=\left\{\frac{\pi}{2}+\frac{\pi}{4}+2 l \pi-\arctan \frac{-B}{A}\right\}$, we can obtain

$$
\begin{aligned}
& \lim _{t \rightarrow \infty} \sup \left(\frac{t^{\rho}}{\rho}\right)^{1-m} \int_{0}^{t}\left(\frac{(t-a)^{\rho}-(w-a)^{\rho}}{\rho}\right)^{\alpha-1} \\
& \quad \times\left(\frac{M^{*}+{ }_{t_{1}} I^{\rho}(g(w) V(w))}{V(w)}\right) \frac{d w}{(w-a)^{1-\rho}}=\infty .
\end{aligned}
$$

Hence, by Theorem 4.1 all the solutions of (5.3) are oscillatory.

Example 5.3 By direct computation, we can find that the function $x(t)=-t^{2}$ is a nonoscillatory solution of problem

$$
\left\{\begin{array}{l}
{ }_{0} \mathfrak{D}^{\frac{3}{2}, 1} x(t)+\sqrt{t}\left(\frac{4}{\sqrt{\pi}}+\frac{e^{\sqrt{x}}}{x^{\frac{1}{4}}}\right)=e^{t}, \quad t>0, \\
\lim _{t \rightarrow 0^{+} 0} \mathfrak{I}^{\frac{1}{2}, 1} x(t)=0 .
\end{array}\right.
$$


Next we will show that condition (3.3) does not hold by setting $\alpha=1 / 2, \rho=1, a=0$, $p(t)=0, q(t)=\sqrt{t}, f(x)=\left((4 / \sqrt{\pi})+\left(e^{\sqrt{x}} / x^{1 / 4}\right)\right), g(t)=e^{t}$, and $V(s)=1$. It is obvious that $(H)$ is satisfied. Therefore, we get

$$
{ }_{t_{1}} I^{1}(g(w) V(w))=e^{w}-e^{t_{1}}
$$

By setting $t_{1}=1$, we obtain

$$
\begin{aligned}
& \left(\frac{t^{\rho}}{\rho}\right)^{1-\alpha} \int_{0}^{t}\left(\frac{(t-a)^{\rho}-(w-a)^{\rho}}{\rho}\right)^{\alpha-1}\left(\frac{M+{ }_{t_{1}} I^{\rho}(g(w) V(w))}{V(w)}\right) \frac{d w}{(w-a)^{1-\rho}} \\
& =2 t^{\frac{1}{2}}\left((M-e) \sqrt{t}+e^{t} \int_{0}^{\sqrt{t}} e^{-s^{2}} d s\right),
\end{aligned}
$$

which yields

$$
\begin{aligned}
& \lim _{t \rightarrow \infty} \inf \left(\frac{t^{\rho}}{\rho}\right)^{1-\alpha} \int_{0}^{t}\left(\frac{(t-a)^{\rho}-(w-a)^{\rho}}{\rho}\right)^{\alpha-1}\left(\frac{M+{ }_{t_{1}} I^{\rho}(g(w) V(w))}{V(w)}\right) \frac{d w}{(w-a)^{1-\rho}} \\
& \quad=\lim _{t \rightarrow \infty} \inf \left\{2 t^{\frac{1}{2}}\left((M-e) \sqrt{t}+e^{t} \int_{0}^{\sqrt{t}} e^{-s^{2}} d s\right)\right\} \\
& \quad=(+\infty) \cdot\left[(M-e)(+\infty)+(+\infty) \frac{\sqrt{\pi}}{2}\right]=\infty .
\end{aligned}
$$

\section{Conclusion}

In this paper force oscillatory properties of solutions of conformable differential equations with damping term are established. The cases of conformable differential equations in the frame of Riemann and Caputo type are considered. A sufficient condition for oscillation of all solutions is given. The obtained results are illustrated by numerical examples. Moreover, a counterexample is presented to show the existence of a nonoscillatory solution in case the conditions do not hold.

\section{Acknowledgements}

The authors express their deep gratitude to the referees for their valuable suggestions and comments for improvement of the paper.

\section{Funding}

A. Aphithana is supported by the Thailand Research Fund through the Royal Golden Jubilee PhD Program (Grant No. PHD/0134/2558).

\section{Availability of data and materials}

Data sharing not applicable to this article as no data sets were generated or analyzed during the current study.

\section{Competing interests}

The authors declare that they have no competing interests.

\section{Authors' contributions}

All authors contributed equally to this work. All authors read and approved the final manuscript.

\section{Author details}

IIntelligent and Nonlinear Dynamic Innovations Research Center, Department of Mathematics, Faculty of Applied Science, King Mongkut's University of Technology North Bangkok, Bangkok, Thailand. '2Department of Mathematics, University of Ioannina, loannina, Greece. ${ }^{3}$ Nonlinear Analysis and Applied Mathematics (NAAM)-Research Group, Department of Mathematics, Faculty of Science, King Abdulaziz University, Jeddah, Saudi Arabia. 


\section{Publisher's Note}

Springer Nature remains neutral with regard to jurisdictional claims in published maps and institutional affiliations.

\section{Received: 11 November 2018 Accepted: 27 February 2019 Published online: 04 March 2019}

\section{References}

1. Miller, K.S., Ross, B.: An Introduction to the Fractional Calculus and Differential Equations. Wiley, New York (1993)

2. Podlubny, I.: Fractional Differential Equations. Academic Press, San Diego (1999)

3. Kilbas, A.A., Srivastava, H.M., Trujillo, J..: Theory and Applications of Fractional Differential Equations. North-Holland Mathematics Studies, vol. 204. Elsevier, Amsterdam (2006)

4. Lakshmikantham, V., Leela, S., Devi, J.V.: Theory of Fractional Dynamic Systems. Cambridge Academic Publishers, Cambridge (2009)

5. Diethelm, K.: The Analysis of Fractional Differential Equations. Lecture Notes in Mathematics. Springer, Berlin (2010)

6. Ahmad, B., Alsaedi, A., Ntouyas, S.K., Tariboon, J.: Hadamard-Type Fractional Differential Equations, Inclusions and Inequalities. Springer, Cham (2017)

7. Agarwal, R.P., Zhou, Y., Wang, J.R., Luo, X.: Fractional functional differential equations with causal operators in Banach spaces. Math. Comput. Model. 54, 1440-1452 (2011)

8. Ahmad, B., Ntouyas, S.K., Alsaedi, A.: New existence results for nonlinear fractional differential equations with three-point integral boundary conditions. Adv. Differ. Equ. 2011, Article ID 107384 (2011)

9. Ahmad, B., Ntouyas, S.K.: Existence results for a coupled system of Caputo type sequential fractional differential equations with nonlocal integral boundary conditions. Appl. Math. Comput. 266, 615-622 (2015)

10. Ahmad, B., Ntouyas, S.K., Tariboon, J.: A study of mixed Hadamard and Riemann-Liouville fractional integro-differential inclusions via endpoint theory. Appl. Math. Lett. 52, 9-14 (2016)

11. Ahmad, B., Alsaedi, A., Aljoudi, S., Ntouyas, S.K.: On a coupled system of sequential fractional differential equations with variable coefficients and coupled integral boundary conditions. Bull. Math. Soc. Sci. Math. Roum. 60(108), 3-18 (2017)

12. Ahmad, B., Ntouyas, S.K.: Existence results for fractional differential inclusions with Erdelyi-Kober fractional integral conditions. An. Ştiinţ. Univ. 'Ovidius' Constanța, Ser. Mat. 25(2), 5-24 (2017)

13. Benchohra, M., Henderson, J., Ntouyas, S.K., Ouahab, A.: Existence results for fractional order functional differential equations with infinite delay. J. Math. Anal. Appl. 338, 1340-1350 (2008)

14. Ambrosio, V:: Zero mass case for a fractional Berestycki-Lions-type problem. Adv. Nonlinear Anal. 7, 365-374 (2018)

15. Denton, Z., Ramírez, J.D.: Existence of minimal and maximal solutions to RL fractional integro-differential initial value problems. Opusc. Math. 37, 705-724 (2017)

16. Cavalcanti, M.M., Domingos Cavalcanti, V.N., Lasiecka, I., Webler, C.M.: Intrinsic decay rates for the energy of a nonlinear viscoelastic equation modeling the vibrations of thin rods with variable density. Adv. Nonlinear Anal. 6 , $121-145$ (2017)

17. Li, W., Radulescu, V.D., Zhang, B.: Infinitely many solutions for fractional Kirchhoff-Schrödinger-Poisson systems. J. Math. Phys. 60, 011506 (2019) 18 pp.

18. Lyons, J.W., Neugebauer, J.T.: Positive solutions of a singular fractional boundary value problem with a fractional boundary condition. Opusc. Math. 37, 421-434 (2017)

19. Chen, D., Qu, P., Lan, Y.: Forced oscillation of certain fractional differential equations. Adv. Differ. Equ. 2013, 125 (2013)

20. Tunc, E., Tunc, O.: On the oscillation of a class of damped fractional differential equations. Miskolc Math. Notes 17 647-656 (2016)

21. Abdalla, B.: Oscillation of differential equations in the frame of nonlocal fractional derivatives generated by conformable derivatives. Adv. Differ. Equ. 2018, 107 (2018)

22. Yang, J., Liu, A., Liu, T.: Forced oscillation of nonlinear fractional differential equations with damping term. Adv. Differ. Equ. 2015, 1 (2015)

23. Grace, S.R., Agarwal, R.P., Wong, P.J.Y., Zafer, A.: On the oscillation of fractional differential equations. Fract. Calc. Appl. Anal. 15, 222-231 (2012)

24. Abdalla, B., Abdeljawad, Th.: On the oscillation of Hadamard fractional differential equations. Adv. Differ. Equ. 2018, 409 (2018)

25. Abdalla, B., Abodayeh, K., Abdeljawad, Th., Alzabut, J.: New oscillation criteria for forced nonlinear fractional difference equations. Vietnam J. Math. 45(4), 609-618 (2017). https://doi.org/10.1007/s10013-016-0230-y

26. Abdalla, B.: On the oscillation of $q$-fractional difference equations. Adv. Differ. Equ. 2017, 254 (2017)

27. Zhou, Y., Ahmad, B., Alsaedi, A.: Existence of nonoscillatory solutions for fractional functional differential equations. Bull. Malays. Math. Sci. Soc. (2017). https://doi.org/10.1007/s40840-017-0511-y

28. Zhou, Y., Alsaedi, A., Ahmad, B.: Oscillation for fractional neutral functional differential systems. J. Comput. Anal. Appl. 25(5), 965-974 (2018)

29. Abdalla, B., Alzabut, J., Abdeljawad, T.: On the oscillation of higher order fractional difference equations with mixed nonlinearities. Hacet. J. Math. Stat. 47, 207-217 (2018)

30. Abdeljawad, T.: On conformable fractional calculus. Comput. Math. Appl. 279, 57-66 (2015)

31. Jarad, F., Abdeljawad, T., Baleanu, D.: On a new class of fractional operators. Adv. Differ. Equ. 2017, 247 (2017) 